\title{
A Smooth Conduit for Electron Fluids
}

\author{
Electrons flow like a viscous fluid through a 2D channel with perfectly \\ smooth sidewalls, offering a new platform to test solid-state and fluid \\ dynamics theories.
}

\section{By Matteo Rini}

ए lectrons can, under certain conditions, flow like a fluid that's thicker than honey. Now researchers have managed to observe this viscous fluid behavior in a way that allows unambiguous measurements and more precise comparisons with theory [1]. Previous studies had demonstrated viscous electron flow in thin channels but could not eliminate the complicated effects of the channel sidewalls. The new experimental setup involves perfectly smooth boundaries and could provide a well-controlled platform for tests of fluid physics and of fundamental theories describing metallic electrons at low temperatures.

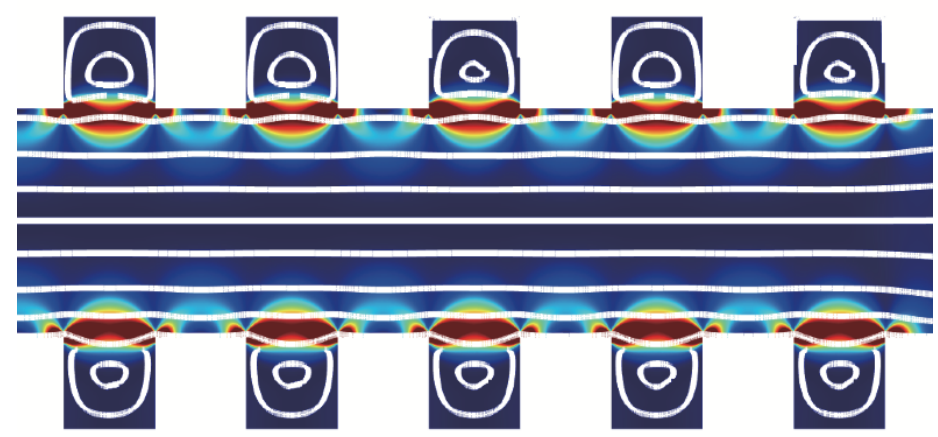

Thicker than honey. This image from a simulation shows streamlines (white) of electrons flowing as a viscous fluid. The loops show that the fluid forms eddies in the crenelles. Colors indicate the power dissipation at each location, from zero (dark blue) to the highest values (dark red).

Credit: A. C. Keser et al. [1]
The textbook theory for electron conduction-the Drude model-describes a solid as a pinball machine, with the electrons bouncing off the atoms in the crystal lattice. But a more detailed theory predicts the possibility of an exotic regime in which the electrons flow through a solid and interact with each other like molecules in a viscous fluid. This fluid-like behavior could be harnessed in novel electronic devices and in experiments that mimic other fluid systems. To reach this regime, the temperature should be so low that electrons are unlikely to scatter from thermally excited crystal vibrations and the material so pure that the scattering of electrons off defects is rare.

In 2016, more than 50 years after the first experimental proposal, electrons in a pristine sheet of the single-molecule-thick material graphene were found to exhibit signatures of a viscous fluid, including the formation of vortices of electric current [2]. A few more studies revealed aspects of viscous fluid behavior in 2D materials, but none of them could precisely characterize the interaction of the electrons with the irregular material boundaries. This "boundary problem" made it difficult to fully model the electron fluid and thus made the observation of viscous flow hard to interpret unambiguously. It also hindered measurements of the fluid's basic properties, including its viscosity.

To solve this problem, Alexander Hamilton of the University of New South Wales in Australia and colleagues used a different approach to create electron channels. In previous devices, the channels were carved in sheets of material using chemical methods, which leave rough sidewalls. In the new scheme, 


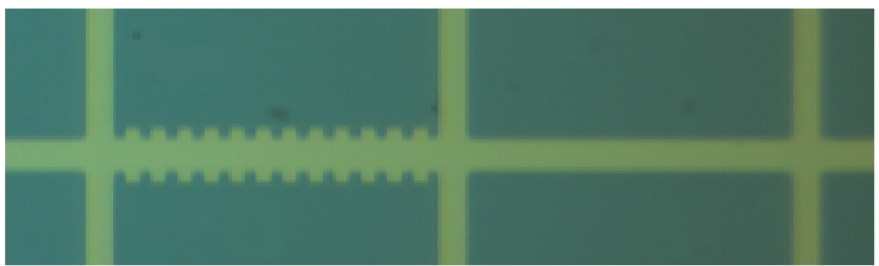

Channeling electrons. Microscope image of two 25-micrometer-long channels used in the experiments. A crenelated channel is left-of-center, and a straight channel is right-of-center.

Credit: A. C. Keser et al. [1]

Hamilton and colleagues produced their channel in the interior of a layered semiconductor. A long and narrow metal electrode atop the layers produced a vertical electric field over a rectangular region that defined the extent of the channel, whose edges lay far away from the physical boundaries of the material. "It's like a motorway with a 'hard shoulder," says Hamilton. The electrons flow through the main lane, without being affected by any "broken pieces" or defects in the emergency lane, he says.

The team found evidence for the smooth boundaries from a comparison between channels of different widths. If there were friction between the electrons and the boundaries, it would increase the channel resistivity (a property related to resistance) as the width decreased, but the resistivity barely changed.

To confirm the viscous flow, the researchers compared the resistance of a straight channel with that of a "crenellated" channel-one whose edges included rectangular crenelles, similar to the top edges of a medieval castle wall. The measured resistance and its dependence on parameters including temperature and magnetic field could only be explained by assuming that the electrons formed eddies within the crenelles.
These eddies are signatures of a viscous fluid. In the absence of sidewall friction, the team could directly determine the electron viscosity from the resistance measurements. They found the electrons to be almost 300 times more viscous than honey.

Hamilton says that this setup could provide an "essentially perfect wind tunnel" for fundamental studies of fluid dynamics and turbulence. A quantitative theory for turbulence is still lacking, and a key obstacle to developing one is designing experiments in which all parameters, including the tunnel's boundary effects, are well controlled. Hamilton envisions embedding objects with various shapes into the electron channel to study the formation of vortices and turbulent flow.

Jean Heremans, who studies charge transport in materials at Virginia Tech in Blacksburg, says that the new scheme may have important implications for solid-state physics. Some basic predictions of Fermi liquid theory-the standard theory for cold, metallic electrons-are difficult to test with high precision because of uncertainties in the measurements, he says. But Hamilton's team was able to use the viscosity measurements to determine, with relatively low uncertainty, a fundamental parameter of the theory: the so-called quasiparticle lifetime. The value was close to, but didn't quite match, the theoretical prediction. Heremans says that the discrepancy has to be further investigated, and having a new, more direct way to gauge quasiparticle lifetime is an "influential development."

Matteo Rini is the Editor of Physics.

\section{REFERENCES}

1. A. C. Keser et al., "Geometric control of universal hydrodynamic flow in a two-dimensional electron fluid," Phys. Rev. X 11, 031030 (2021).

2. L. Levitov and G. Falkovich, "Electron viscosity, current vortices and negative nonlocal resistance in graphene," Nat. Phys. 12 (2016). 